\title{
Research priorities in bronchiectasis: a consensus statement from the EMBARC Clinical Research Collaboration
}

\author{
Stefano Aliberti ${ }^{1}$, Sarah Masefield ${ }^{2}$, Eva Polverino ${ }^{3}$, Anthony De Soyza ${ }^{4,5}$, \\ Michael R. Loebinger ${ }^{6}$, Rosario Menendez ${ }^{7}$, Felix C. Ringshausen ${ }^{8}$, \\ Montserrat Vendrell ${ }^{9}$, Pippa Powell ${ }^{2}$ and James D. Chalmers ${ }^{10}$ on behalf of the \\ EMBARC Study Group ${ }^{11}$
}

\begin{abstract}
Affiliations: ${ }^{1}$ Dept of Pathophysiology and Transplantation, University of Milan, Cardio-thoracic unit and Adult Cystic Fibrosis Center, Fondazione IRCCS Cà Granda Ospedale Maggiore Policlinico, Milan, Italy. ${ }^{2}$ European Lung Foundation, Sheffield, UK. ${ }^{3}$ Fundaciò Clìnic, IDIBAPS, CIBERES, Hospital Clinic de Barcelona, Barcelona, Spain. ${ }^{4}$ Institute of Cellular Medicine, Newcastle University, Newcastle upon Tyne, UK. ${ }^{5}$ Bronchiectasis Service, Freeman Hospital, Newcastle upon Tyne, UK. ${ }^{6}$ Host Defence Unit, Royal Brompton Hospital, London, UK. ${ }^{7}$ Pneumology Service, Universitary and Polytechnic Hospital La Fe, Valencia, Spain. ${ }^{8}$ Dept of Respiratory Medicine, Hannover Medical School, Member of the German Center for Lung Research (DZL), Hannover, Germany. ${ }^{9}$ Bronchiectasis Group, Girona Biomedical Research Institute (IDIBGI), Dr. Trueta University Hospital, Girona, Spain. ${ }^{10}$ College of Medicine, University of Dundee, Dundee, UK. ${ }^{11}$ For a list of the EMBARC Study Group investigators see the Acknowledgements section.
\end{abstract}

Correspondence: Stefano Aliberti, Dept of Pathophysiology and Transplantation, University of Milan, Cardiothoracic unit and Adult Cystic Fibrosis Center, Fondazione IRCCS Cà Granda Ospedale Maggiore Policlinico, Via F. Sforza 35, Milan, Italy. E-mail: stefano.alibertiđunimi.it

ABSTRACT Bronchiectasis is a disease of renewed interest in light of an increase in prevalence and increasing burden on international healthcare systems. There are no licensed therapies, and large gaps in knowledge in terms of epidemiology, pathophysiology and therapy. The European Multicentre Bronchiectasis Audit and Research Collaboration (EMBARC) is a European Respiratory Society (ERS) Clinical Research Collaboration, funded by ERS to promote high-quality research in bronchiectasis. The objective of this consensus statement was to define research priorities in bronchiectasis. From 2014 to 2015, EMBARC used a modified Delphi process among European bronchiectasis experts to reach a consensus on 55 key research priorities in this field. During the same period, the European Lung Foundation collected 711 questionnaires from adult patients with bronchiectasis and their carers from 22 European countries reporting important research priorities from their perspective. This consensus statement reports recommendations for bronchiectasis research after integrating both physicians and patients priorities, as well as those uniquely identified by the two groups. Priorities identified in this consensus statement provide the clearest possible roadmap towards improving our understanding of the disease and the quality of care for patients with bronchiectasis.

@ERSpublications

EMBARC consensus statement identifies research priorities in bronchiectasis as determined by physicians and patients http://ow.ly/lmcj300a2iS

Received: Nov 122015 | Accepted after revision: March 02 2016 | First published online: June 102016

This Task Force document was endorsed by the ERS Science Council and ERS Executive Committee in April 2016.

This article has supplementary material available from erj.ersjournals.com

Support statement: EMBARC is funded by the Inhaled Antibiotics in Bronchiectasis and Cystic Fibrosis consortium, a project of the European Union Innovative Medicines Initiative (number 115721), and by the European Respiratory Society as Clinical Research Collaboration. James D. Chalmers acknowledges fellowship support from the Wellcome Trust. Funding information for this article has been deposited with the Open Funder Registry.

Conflict of interest: S. Masefield and P. Powell are employees of the European Lung Foundation. Further disclosures can be found alongside this article at erj.ersjournals.com

Copyright OERS 2016 


\section{Introduction}

Bronchiectasis is a chronic lung disorder characterised by permanent dilatation of bronchi leading to impaired mucociliary clearance, chronic airway inflammation and bacterial colonisation [1]. Although historically considered a neglected disease, bronchiectasis has become a disease of renewed interest over the past decades in light of an increase in prevalence and an increasing burden on healthcare systems [2-8]. To date, treatment of bronchiectasis is mainly extrapolated from cystic fibrosis (CF) and chronic obstructive pulmonary disease (COPD), or based on expert opinions as high-quality evidence is still missing [9]. Large gaps in our knowledge could be identified on several aspects of this disease and this emphasises the need for additional clinical and translational research, as well as collaborative working.

Towards these goals, the European Multicentre Bronchiectasis Audit and Research Collaboration (EMBARC) was developed in 2012 as the first international bronchiectasis network, seeking to promote clinical and

\section{BOX 1 Summary of recommendations}

1) DNA biobanks linked to well-phenotyped patient cohorts should be established to enable underlying genetic susceptibility to bronchiectasis to be established.

2) Observational research in large patient cohorts is needed to establish the natural history of bronchiectasis due to different aetiologies.

3) A comprehensive study enrolling patients when stable and during exacerbation should be conducted, evaluating the impact of bacteria, viruses, fungi and noninfectious stimuli to identify the cause(s) of bronchiectasis exacerbations.

4) Studies are required to optimise compliance, and access to chest physiotherapy and pulmonary rehabilitation in bronchiectasis.

5) A deeper understanding of the inflammatory pathways in bronchiectasis is needed to develop new therapies. We recommend using emerging techniques and technologies (particularly proteomics, metabolomics and genomics) in large, well-characterised cohorts to identify new treatment targets and deeper patient phenotyping.

6) An implementation study should be performed to investigate whether the use of bronchiectasis severity scores could improve patient care.

7) A randomised controlled trial of Pseudomonas aeruginosa eradication therapy, compared to no eradication treatment, should be performed.

8) A randomised controlled trial comparing at least 14 days of antibiotic treatment for exacerbations with shorter-course treatments is required.

9) We suggest studies of the microbiome (incorporating bacteria and potentially fungi) in bronchiectasis linked to detailed clinical phenotyping data.

10) A longitudinal study of the bacteriology of bronchiectasis incorporating data on antibiotic resistance is needed.

11) Longitudinal studies should be conducted in patients receiving oral and inhaled antibiotics to monitor for the emergence of antibiotic resistance.

12) Studies should ideally evaluate whether cyclical or continuous administration of long-term antibiotics is superior both in terms of clinical efficacy and the emergence of resistance.

13) Further studies are required to define the optimal patient population to benefit from long-term macrolide therapy.

14) More "real world" data on the long-term safety and resistance impact of macrolide treatment are required.

15) Inhaled antibiotics such as colistin, gentamicin and tobramycin should be subject to definitive phase III trials to demonstrate a reduction in exacerbations and improvements in quality of life.

16) Mechanistic studies investigating the genetic, microbiological, inflammatory and clinical susceptibility factors for $P$. aeruginosa colonisation should be conducted.

17) Long-term cohort studies are needed to identify which patients acquire $P$. aeruginosa colonisation and to identify its independent effects on outcome.

18) Comparative studies are needed to determine the optimal choice between oral and inhaled antibiotic treatment in patients with and without $P$. aeruginosa colonisation.

19) Randomised controlled trials should address whether alternative long-term oral antibiotics lother than macrolides) are effective at reducing exacerbations.

20) Studies should be conducted to determine the effectiveness of patient self-management in bronchiectasis and adherence to treatment.

21) Further research with patients as partners could explore the specific information needs of bronchiectasis patients, effective health care professionals and patient communication strategies, and develop improved patient-reported outcomes.

22) A multidisciplinary education programme is needed for bronchiectasis to increase awareness among non-specialists in secondary care and among primary care. 
translational research in bronchiectasis (www.bronchiectasis.eu) [10]. Since 2014, the EMBARC network also represents an official European Respiratory Society (ERS) Clinical Research Collaboration (CRC), a group of researchers approved and funded by the ERS to promote collaboration and research excellence in Europe and beyond. With this ERS support has come the additional benefit of patient involvement facilitated by the European Lung Foundation (ELF) (www.europeanlung.org). The ELF was founded in 2000 with the aim of bringing together patients and the public with respiratory professionals to positively influence lung health. One way that this is achieved is by involving patients in identifying patient-centred outcomes and a focus on quality of life integrated into the EMBARC and CRC activities [11].

The first challenge the EMBARC collaborative group has recognised was to reach a consensus on the main clinical and translational research priorities in the field of bronchiectasis. We used a modified Delphi process among European bronchiectasis experts alongside a questionnaire of European patients with bronchiectasis and carers to identify the major clinical and research priorities in this disease. Here, we present a consensus statement of the EMBARC CRC based on the findings of these survey approaches.

\section{Methods}

Two parallel processes were performed: a modified Delphi process involving European physicians caring for patients with bronchiectasis and a questionnaire process to survey the perspectives of patients with bronchiectasis and carers (figure 1).

\section{Experts' research priorities}

From July 2014 to November 2014, a working group composed of eight EMBARC members from five European countries was established. The objective of this group was to systematically evaluate the available literature and to produce a consensus on the most important research questions in the field of bronchiectasis. A systematic review was conducted to ask research questions on adult non-CF bronchiectasis suggested by authors of editorials, letters, narrative reviews, meta-analysis, guidelines and original articles published from 2010 to 2014 in English. The following keyword was selected as part of the title in Pubmed: "bronchiectasis". The search retrieved 448 manuscripts that were reviewed by the committee in order to generate research questions in bronchiectasis, supplemented by expert opinion. After that, the working group was asked to draft the most important research questions on bronchiectasis, split into 10 separate sections: epidemiology; pathogenesis and mechanisms of the disease; aetiology, radiology and physiology; microbiology and microbial diagnostics; acute and long-term suppressive antibiotic therapy; nonantibiotic and anti-inflammatory therapies; physiotherapy and pulmonary rehabilitation; outcomes, prognosis and healthcare utilisation; exacerbation; and others. A Delphi approach was then used to reach a consensus (agreement $>85 \%$ ) on the most important research questions in the field [12]. The process was carried out anonymously. From December 2014 to February 2015, the list of research priorities agreed by the working group was sent electronically to 138 EMBARC members representing 23 European

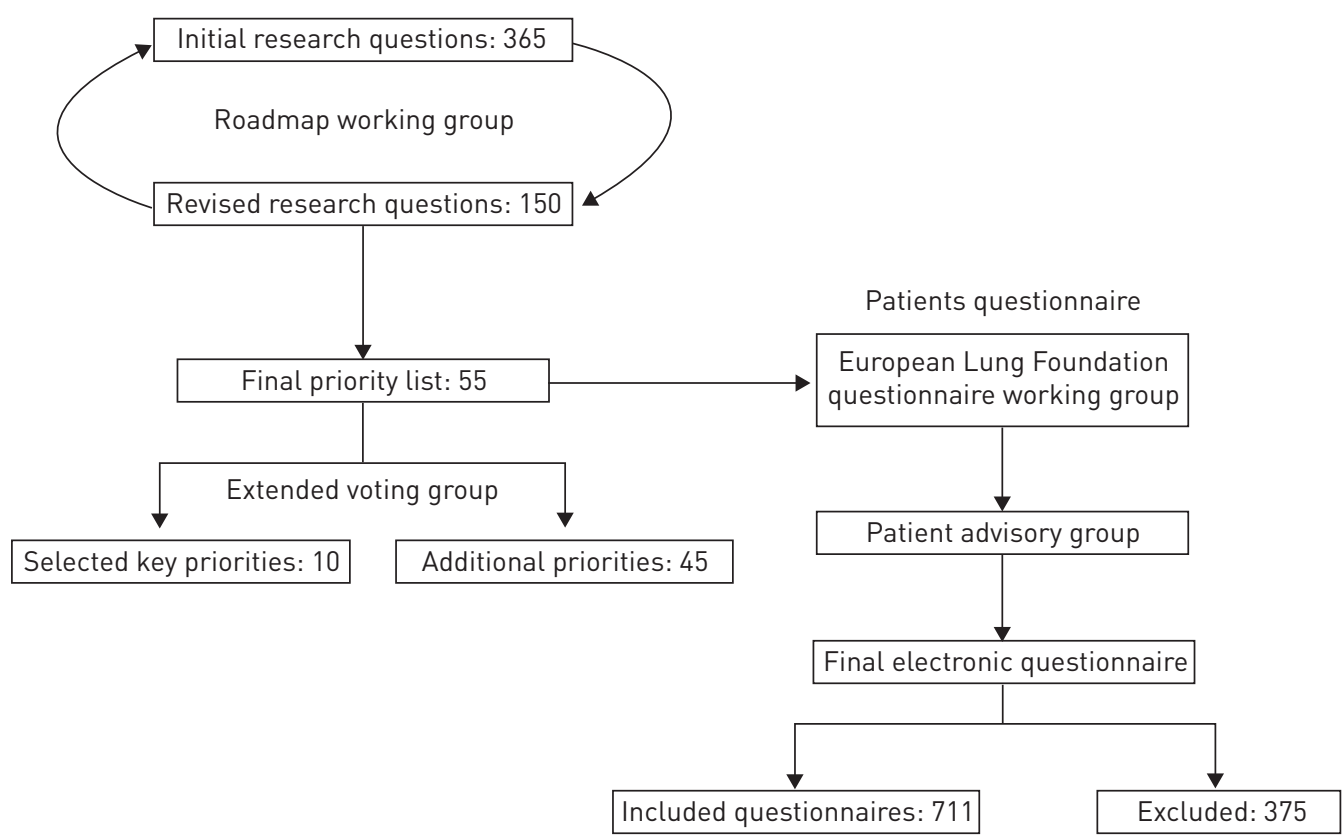

FIGURE 1 Processes to define both experts' and patients' research priorities in bronchiectasis. 
countries who were asked to grade each research question anonymously using a five-level scale (from unimportant to essential).

\section{Patients' and carers' research priorities}

During the same period, a questionnaire to look into the research priorities of patients and carers was developed. The aim was to identify the challenges of treating and living with bronchiectasis from the perspectives of people with bronchiectasis, their families and friends, and to prioritise what they think needs to change or be considered to have the greatest impact on quality of life for people with bronchiectasis. The questionnaire was developed by members of the EMBARC Roadmap Study Group (S. Aliberti, J.D. Chalmers and E. Polverino) and ELF (S. Masefield and P. Powell), reviewed by an advisory group of four "expert patients" with bronchiectasis, and revised to ensure that it met the needs of the project and would be relevant, interesting and accessible for patients and carers (online supplementary material). Furthermore, the items in the questionnaire were broadly aligned with the 10 research sections identified by the experts to be covered in the roadmap so that the relative significance for patients and professionals could be compared. Expert questions were translated into language accessible to patients by the advisory group, and in some cases expert questions were discarded or replaced. The respondents were asked to rate anonymously what aspect of their or their relative/friend's bronchiectasis is found the most difficult to manage. For each aspect listed, they could select "not an issue", "not very difficult", "difficult", "very difficult" or "no opinion". Respondents were also asked to prioritise questions according to four areas of research (bronchiectasis management by doctors; treatment; monitoring; and self-management by patients), selecting "unimportant", "not very important", "important", "very important" or "no opinion" for each of them. The questionnaire was published online, and an invitation was sent to patients with bronchiectasis all over Europe via ERS, ELF and EMBARC patient and healthcare professional (HCP) networks/clinics. The questionnaire was available online from December 2014 to April 2015, in 12 languages (English, German, French, Dutch, Spanish, Italian, Portuguese, Greek, Russian, Polish, Turkish and Arabic). The questionnaire was also available in a printable format for HCPs to provide to their patients with a paper copy, with the responses input online anonymously by a member of administrative staff at the HCP's clinic. Ethical approval was waived for the active involvement of patients as either advisors or participants in questionnaires (online supplementary material).

\section{Consensus statements}

Following review of the highest scoring research priorities across both physician and patient survey exercises, the EMBARC roadmap working group has produced a series of agreed consensus statements on research priorities in bronchiectasis.

\section{Results}

\section{Experts' research priorities}

A total of 365 potential research questions were initially drafted by the EMBARC Roadmap Study Group and after a check for repetitions, discrepancy or inconsistency, a shortlist of 150 research questions was defined. The modified Delphi process then involved several rounds of revision, in which the study group agreed with, disagreed with or suggested changes to the 150 research questions that they and the other participants had proposed. The responses were collated and sent back to participants who were then able to revise their judgment in light of the group feedback. This process was repeated three times. Response from the members of the EMBARC Roadmap Study Group was 100\% during each of the three rounds. At the end of the Delphi process, a final consensus ( $>85 \%$ agreement) was reached on 55 research questions. To determine which of these research questions had the greatest priority, the 55 research questions were then sent electronically to 138 EMBARC members representing 23 European countries. 100 EMBARC members graded the list of research questions (response rate $72 \%$ ). The final list of the experts' research priorities after grading is shown in table 1.

\section{Patients' research priorities}

A total of 1086 questionnaires were completed. Of these, 711 were considered for analysis, meeting the following criteria: age, sex, resident in geographical Europe, and either a person with bronchiectasis, or a parent, relative, carer or friend of someone with bronchiectasis. The respondents covered 22 countries (UK, Germany, Turkey, Spain, France, Italy, Portugal, the Netherlands, Russia, Ireland, Belgium, Norway, Greece, Switzerland, Poland, Hungary, Bulgaria, Romania, Austria, Sweden, Finland and Luxembourg) (supplementary figure S1). The dominant characteristics of respondents were: $87 \%$ patients; $71 \%$ female; $30 \%$ aged $31-50$ years; and $27 \%$ aged $61-70$ years (figures S2 and S3).

The aspects of bronchiectasis that patients considered the most difficult to manage are reported in figure $2 \mathrm{a}$. Every aspect listed was found difficult by $>23 \%$ of respondents. However, despite all aspects being found 
TABLE 1 Ranking of experts' research priorities in bronchiectasis

Research priority

When and how (molecule, dose, regimen, route (intravenous, oral or inhaled/nebulised) and duration) should Pseudomonas aeruginosa be eradicated in patients with bronchiectasis, and do patients' outcomes improve after that?

2

What are the indications and what is the optimal antibiotic therapy (dosage, how many antibiotics, type, oral versus intravenous versus inhaled/nebulised and length of therapyl for an exacerbation of bronchiectasis?

3

What are the prevalence and characteristics of microbiological colonisation, and chronic and acute infections (exacerbations and pneumonial in patients with bronchiectasis across Europe (including bacteria, viruses, fungi, nontuberculous mycobacteria and resistant microorganisms)?

4

What are the risk factors and causes of fast progression and poor outcomes (e.g. hospitalisation, lung transplantation and mortality) in patients with bronchiectasis?

5

What is the impact of long-term antibiotic therapy on microbial resistances?

6

When should a long-term suppressive antibiotic therapy leither oral or inhaled/nebulised) be started in patients with bronchiectasis laccording to the presence or absence of $P$. aeruginosa or other pathogens) and what would be end-points for efficacy?

$7 \quad$ What are the key factors leading to $P$. aeruginosa colonisation?

8 What are the indications of oral versus inhaled/nebulised long-term suppressive antibiotic treatment?

$9 \quad$ What are the best molecule, dose, regimen and duration for long-term oral antibiotic therapy in patients with bronchiectasis laccording to the presence or absence of $P$. aeruginosa or other pathogens)?

What are the causes of an exacerbation of bronchiectasis?
When and how (molecule, dose, regimen, route lintravenous, oral or inhaled/nebulised) and duration) should pathogens other than $P$. aeruginosa be eradicated in patients with bronchiectasis, and do patients' outcomes improve after that?

What are the best molecule, dose, regimen and duration for long-term inhaled/nebulised antibiotic therapy in patients with bronchiectasis laccording to the presence or absence of $P$. aeruginosa or other pathogens)?

What are the baseline investigations to evaluate aetiologies in patients with bronchiectasis?

Do different aetiologies of bronchiectasis predetermine microbiological characteristics, and affect severity, patients' quality of life and disease progression in patients with bronchiectasis?

How should the severity of an exacerbation of bronchiectasis be assessed and what is its impact on long-term outcomes?

When should airway drainage techniques be started in patients with bronchiectasis, and which one is the most effective and pragmatic?

Which is the most useful severity score in clinical practice in patients with bronchiectasis?

Does an early referral to a specialist clinic change outcomes in patients with bronchiectasis?

What is the average lung function decline in patients with bronchiectasis across Europe and what are the risk or protective factors for that?

Which factors, including aetiology of bronchiectasis, patients' characteristics or bacteria isolated in sputum, affect macrolide efficacy in patients with bronchiectasis?

What are the characteristics of patients' microbiomes, both during the stable state and exacerbation, and what is its impact on severity of the disease and follow-up?

What is the role of viruses, atypicals, fungi and anaerobes (both singly and in co-infection) in patients with bronchiectasis, during both the stable state and exacerbation, and what is their impact of patients' severity and outcomes?

\section{Area of research}

Score

Acute and long-term suppressive antibiotic therapy

$4.35 / 5$

Exacerbation

Epidemiology

Outcomes, prognosis and

healthcare utilisation

Acute and long-term suppressive antibiotic therapy

Acute and long-term suppressive antibiotic therapy

Microbiology and microbial diagnostics

Acute and long-term suppressive antibiotic therapy

Acute and long-term suppressive antibiotic therapy

Exacerbation

Acute and long-term suppressive antibiotic therapy

Acute and long-term suppressive antibiotic therapy

Aetiology, radiology and pulmonary function tests

Aetiology, radiology and pulmonary function tests

Physiotherapy and pulmonary rehabilitation

Outcomes, prognosis and healthcare utilisation

Outcomes, prognosis and healthcare utilisation

Aetiology, radiology and pulmonary function tests

Acute and long-term suppressive antibiotic therapy

Microbiology and microbial diagnostics

Microbiology and microbial diagnostics 
TABLE 1 Continued

\section{Research priority}

23

24

25 Europe? bronchiectasis? Europe? bronchiectasis? bronchiectasis? bronchiectasis?
What is the prevalence of different aetiologies of bronchiectasis across

Are influenza and/or pneumococcal vaccines and other immunotherapies effective in preventing exacerbations in patients with bronchiectasis?

What are the adverse events of a both oral and inhaled/nebulised suppressive antibiotic therapy in patients with bronchiectasis?

What are the prevalence and type of long-term suppressive oral (macrolide and nonmacrolide) and nebulised/inhaled antibiotic therapy in patients with bronchiectasis across Europe?

When should pulmonary rehabilitation be started in patients with bronchiectasis and what is its impact on patients' outcomes?

What are incidence, prevalence, patients' demographic characteristics and comorbidities of bronchiectasis across Europe?

Is airway clearance useful during an acute exacerbation of bronchiectasis?

Should we establish new breakpoints for predicting bacteria susceptibility when inhaled/nebulised antibiotics are used?

What are the indications/contraindications for lung transplantation in bronchiectasis and what are patients' outcomes after lung transplantation?

Which are the best systemic (e.g. blood) or local (e.g. sputum) inflammatory markers for the diagnosis, management and follow-up of patients with bronchiectasis?

What is the distribution of inhaled/nebulised antibiotics with different formulations and devices in lungs with bronchiectasis?

Do specific patient education packages, self-management plans and patients support groups improve outcomes in patients with

Where are patients with bronchiectasis managed across Europe, including specialist (bronchiectasis) clinics, CF centres/clinics, respiratory clinics or general practitioners?

Are other functional tests (such as carbon monoxide diffusing capacity, 6-min walk test, lung clearance index, endurance shuttle walk, incremental exercise tests or accelerometers) markers for severity of the disease, outcomes and end-points for the clinic?

What is the best approach/score to evaluate radiological severity in patients with bronchiectasis?

Might cross-infection occur in patients with bronchiectasis and is patients segregation required?

What are the healthcare costs of bronchiectasis management across

What is the role of inhaled hyperosmolar therapy le.g. hyaluronate; mannitol; or $\mathrm{NaCl} 3 \%, 6 \%$ or $7 \%)$ ?

What are the radiological changes in bronchiectasis over time?

What is the impact of haemoptysis on the prognosis of patients with bronchiectasis and how should it be managed?

What are the genetic and epigenetic findings in patients with bronchiectasis compared to healthy controls, and what is their role in acquisition of specific pathogens and patients' outcomes?

What are the characteristics and outcomes of patients with bronchiectasis undergoing surgery, including segmentectomy, lobectomy or pneumonectomy?

What is the role of systemic steroids during an exacerbation of

Is there an increased rate of innate immune defects (e.g. mannose-binding lectin deficiency, common variable immunodeficiency, IgM or IgA deficiency, or complement deficiencyl in specific patients with

What is the role of long-term inhaled corticosteroids in patients with

What are the frequency of CF heterozygosity, and the role of CFTR and $\mathrm{ENaC}$ dysfunction in patients with bronchiectasis?

\section{Area of research}

Score

Epidemiology

$3.87 / 5$

Other

Acute and long-term suppressive

antibiotic therapy

Epidemiology

Physiotherapy and

pulmonary rehabilitation

Epidemiology

Exacerbation

$3.80 / 5$

Microbiology and microbial diagnostics

Other

$3.73 / 5$

Pathogenesis and mechanisms of the disease

Acute and long-term

ther

Epidemiology

Aetiology, radiology and pulmonary function tests

Aetiology, radiology and pulmonary function tests

Epidemiology

Nonantibiotic and anti-inflammatory therapies

Aetiology, radiology and pulmonary function tests Other

Pathogenesis and mech anisms of the disease

Other

Exacerbation

Pathogenesis and mechanisms of the disease

Nonantibiotic and anti-inflammatory

Pathogenesis and mechanisms of the disease 
TABLE 1 Continued

Research priority

Area of research

Score

49 What is the role of oral mucolytics in patients with bronchiectasis?

Nonantibiotic and

$3.35 / 5$

anti-inflammatory therapies

50 What is the role of

antiproteinases/elastase inhibitors in patients with bronchiectasis?

Nonantibiotic and

$3.19 / 5$

anti-inflammatory therapies

$3.15 / 5$

nonsteroidal anti-inflammatory drugs) during the stable

Nonantibiotic and

state in patients with bronchiectasis?

52

What is the role of protease-antiprotease imbalance, matrix anti-inflammatory therapies

Pathogenesis and metalloproteinase and neuron-specific enolase

mechanisms of the disease

in patients with bronchiectasis?

53

What is the role of anticholinergic therapy in patients with bronchiectasis?

Nonantibiotic and

anti-inflammatory therapies

$54 \quad$ What is the role of phosphodiesterase type 4 inhibitors

Nonantibiotic and

in patients with bronchiectasis?

55

Does daily proton-pump inhibitor use impact clinical outcomes in patients with bronchiectasis?

anti-inflammatory therapies

Nonantibiotic and

anti-inflammatory therapies

CF: cystic fibrosis; CFTR: cystic fibrosis transmembrane conductance regulator; ENaC: epithelial sodium channel.
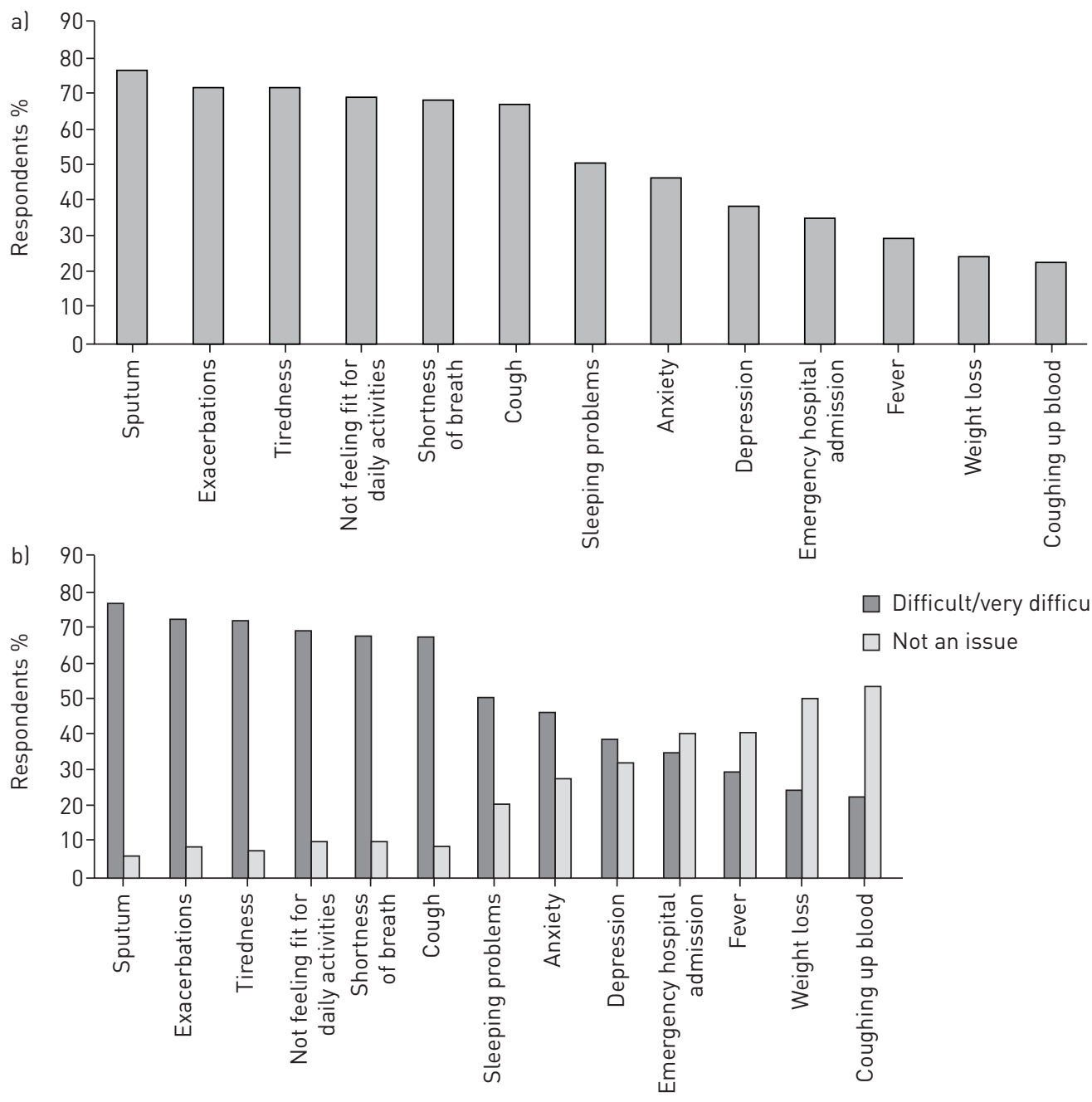

FIGURE 2 a) Aspects of bronchiectasis found either difficult or very difficult by patients. b) Aspects of bronchiectasis found difficult or very difficult by patients in comparison with the percentage of patients who identified the same aspects as "not an issue". 
difficult or very difficult by some, seven of the aspects were also identified as not an issue for between $21 \%$ and $53 \%$ of respondents (figure $2 \mathrm{~b}$ ). Thus, each person's experience of their bronchiectasis and the aspects that they find most difficult to manage varies.

Patients' research priorities are reported in figures S4-S7 of the online supplementary material, according to the four research areas (bronchiectasis management by doctors; treatment; monitoring; and self-management by patients), while table 2 shows the 29 research priorities across all the four areas. In addition, $42 \%$ of

TABLE 2 Ranking of patients' and carers' research priorities in bronchiectasis

\section{Research priority}

How does bronchiectasis develop and continue?

How can communication between healthcare professionals and each patient be optimised to improve self-management?

3

5

6

7

8

What makes some patients get worse?

What are causes of bronchiectasis?

What are the triggers for an exacerbation?

How can self-management programmes and care plans designed with each person be most effective in helping patients have greater control over their condition and recognise/manage an exacerbation?

How can physiotherapy be accessible to all patients, and teach them to use the techniques and how to use the equipment at home effectively?

How can reliable, plain language information on living with bronchiectasis be accessible to patients?

How can patients at increased risk of poor outcomes or needing urgent treatment be identified?

How can awareness of bronchiectasis in community care services be improved (e.g. among community-based nurses and physiotherapists)?

Are there ways to diagnose bronchiectasis earlier?

How can awareness of the role of physiotherapy and pulmonary rehabilitation in treating bronchiectasis be improved?

Can we test new techniques for managing bronchiectasis in real-world environments, such as at home and community settings (not in the laboratory or in hospitals), to improve how bronchiectasis is managed?

Is there a link between getting a cold and exacerbation?

Can regular lung function testing help notice changes or increased risk of an exacerbation?

Is there a relationship between bronchiectasis and other conditions, such as asthma, "acid" reflux and inflammatory bowel diseases?

Can new medicines that can be taken in new ways be developed (e.g. inhaled or nebulised)?

Can regular sputum examinations when a person is stable and during exacerbation help us learn more about how the condition changes?

Can we develop better ways of teaching people to use their medicines?

Can we provide and will providing test results help each patient follow their progress?

How can primary care doctors be educated to prescribe the same dose/length of antibiotic therapy for exacerbations in bronchiectasis as used in CF?

Can vaccines be developed/used to prevent exacerbations?

How often/why does bronchiectasis occurs in certain groups of people?

How can we ensure that each person has access to a home intravenous antibiotic service to avoid unnecessary hospital admissions?

How can awareness, and use of peer support forums and social media to exchange information be raised?

How can patients have and use equipment at home to monitor their symptoms?

How can the monitoring and treatment of coughing up blood be achieved?

Can using longer-term antibiotic therapy when a person's condition is stable improve treatment?

Can having regular computed tomography scans to look for changes and increased risk of an exacerbation improve monitoring?

\section{Area of research}

How bronchiectasis is managed by doctors Self-management by patients

How bronchiectasis is managed by doctors How bronchiectasis is managed by doctors How bronchiectasis is managed by doctors Self-management by patients

Self-management by patients

Self-management by patients

How each person's bronchiectasis is monitored

How bronchiectasis is treated

How bronchiectasis is managed by doctors How bronchiectasis is treated

How bronchiectasis is managed by doctors

How bronchiectasis is managed by doctors How each person's bronchiectasis is monitored

How bronchiectasis is managed by doctors

How bronchiectasis is treated

How each person's bronchiectasis is monitored

How bronchiectasis is treated

Self-management by patients

How bronchiectasis is treated

How bronchiectasis is treated

How bronchiectasis is managed by doctors

Self-management by patients

Self-management by patients

How each person's bronchiectasis is monitored

How each person's bronchiectasis is monitored How bronchiectasis is treated

How each person's bronchiectasis is monitored

CF: cystic fibrosis. 
respondents outlined other important topics for research, with the most common additional themes being infection and the immune system, mental health and quality of life, nutrition and exercise, and managing mucus/phlegm.

The key research priorities are now briefly discussed in the following sections: 1) research priorities commonly identified by both experts and patients; 2) important research priorities identified by experts; and 3) important research priorities identified by patients. The full version of the discussion of research priorities is reported in the online supplementary material.

\section{Discussion}

Research priorities commonly identified by both experts and patients

What are the causes of bronchiectasis? (Patients)

What are the baseline investigations to evaluate aetiologies in patients with bronchiectasis? (Experts)

One of the cornerstones of the management of bronchiectasis is the identification and treatment of underlying causes. Several predisposing factors might be identified including previous severe respiratory infections, allergic bronchopulmonary aspergillosis, impairment of ciliary clearance, primary or secondary immunodeficiency, and other diseases associated with bronchiectasis, such COPD and severe asthma. Despite following guideline recommendations, an aetiology of bronchiectasis cannot be ascertained in $40 \%$ of patients, whilst an aetiology of bronchiectasis leading to a change in patients' management may be identified in only $13 \%$ of the cases [13]. Further research should integrate basic research from the "-omics" perspective with clinical data in order to identify the possible aetiologies among the large group of patients with idiopathic bronchiectasis.

\section{Consensus statements}

1) DNA biobanks linked to well-phenotyped patient cohorts should be established to enable underlying genetic susceptibility to bronchiectasis to be established.

2) Observational research in large patient cohorts is needed to establish the natural history of bronchiectasis due to different aetiologies.

What are the triggers of an exacerbation? (Patients)

What are the causes of an exacerbation of bronchiectasis? (Experts)

From an infective point of view, changes in airway bacterial community composition, emergence of new strains and spread of infection by the same species to new regions of the lung might trigger exacerbations [14-16]. New airway infection caused by organisms present in low abundance (and thus, that may not be detected by conventional techniques) yet identifiable by metagenomic approaches could help us in understanding what is responsible for triggering a new exacerbation [17]. It could be also possible that exacerbations may be driven by changes and/or adaptation in strains that cannot be detected by such approaches, and hence, metabolomic techniques may help. The role of viruses in triggering infective exacerbations should also be better defined [18], as well as their interaction with bacteria in both the stable state and during exacerbations. Finally, the "vicious cycle" hypothesis that characterised the physiopathology of bronchiectasis patients in the stable state does not rule out the possibility that noninfectious triggers, including indoor and outdoor air pollution, might cause exacerbations and further prospective observational studies are needed in this area [19].

\section{Consensus statement}

A comprehensive study enrolling patients when stable and during exacerbation should be conducted, evaluating the impact of bacteria, viruses, fungi and noninfectious stimuli to identify the cause(s) of bronchiectasis exacerbations.

\section{How can we improve the access to physiotherapy and home-use techniques? (Patients) \\ When should airway drainage techniques be started in patients with bronchiectasis, and which one is the most effective and pragmatic? (Experts)}

Interventions aimed at promoting clearance of excess mucus are a mainstay of bronchiectasis management [1]. Although few studies have explored the impact of physiotherapy in bronchiectasis, airway clearance techniques seem to be safe and allow a better sputum expectoration with an increase in patients' quality of life [20]. Evidence on pulmonary rehabilitation is scarce; however, most of the studies demonstrated an increase in patients' performance and quality of life, and an increase of the time to the next exacerbation [21-23]. Both physicians and patients agreed that additional controlled trials of these interventions would be beneficial but that the priority may be in identifying methods that are accessible and that encourage adherence. 
Consensus statement

Studies are required to optimise compliance and access to chest physiotherapy and pulmonary rehabilitation in bronchiectasis.

Identify patients at risk of poor outcomes (Patients)

What are the risk factors and causes for fast progression and poor outcomes in patients with bronchiectasis? (Experts)

Two scores have been recently proposed to predict adverse outcomes in bronchiectasis: the Bronchiectasis Severity Index (BSI) and the FACED (forced expiratory volume in $1 \mathrm{~s}$, age, colonisation with Pseudomonas aeruginosa, radiological extent and dyspnoea) score $[4,24]$. The BSI has been shown to accurately identify not only patients at risk of death, but also those with the highest risk of complications, including exacerbations, hospitalisation and impaired quality of life. Expert opinion suggests that disease severity may be useful as a framework for clinical decisions, allowing the appropriate targeting of therapies including long-term macrolides, inhaled antibiotic treatment and airway adjuncts [1]. An accurate assessment of prognosis is also essential for rational decisions regarding transplantation in bronchiectasis, and scoring may also be helpful in this context [25]. Several other factors need future multicentre, prospective, longitudinal studies to evaluate drivers of faster disease progression, including the evaluation of microorganisms other than $P$. aeruginosa, microbiome parameters such as species diversity and richness, local and systemic inflammatory biomarkers, and other measurements of lung function impairment. The importance of comorbidities should also be explored as they may be amenable to treatment [26].

Consensus statements

1) A deeper understanding of the inflammatory pathways in bronchiectasis is needed to develop new therapies. We recommend using emerging techniques and technologies (particularly proteomics, metabolomics and genomics) in large, well-characterised cohorts to identify new treatment targets and deeper patient phenotyping.

2) An implementation study should be performed to demonstrate if the use of bronchiectasis severity scores could improve patient care.

Important research priorities identified by experts

When and how should P. aeruginosa be eradicated in patients with bronchiectasis and does eradication result in improved outcomes?

$P$. aeruginosa colonisation defines a specific clinical phenotype of bronchiectasis, and is associated with a three-fold increase risk of death, a nearly seven-fold increase risk of hospital admissions, worse quality of life and more frequent exacerbations [27-29]. Evidence from CF suggests that attempts at eradication therapy targeting Pseudomonas can have success in converting patients to culture-negative status [30]. Data in bronchiectasis and CF are of limited quality in defining both the early outcomes and long-term benefits. There are no large, adequately powered studies to inform current practice, with most studies limited to observational case series [31,32]. Other studies using inhaled antibiotics therapies focused on treating those with persistent infection, with the primary aim of reducing exacerbations [33]. An unexpected benefit seen in these trials is that they have consistently demonstrated small but significant rates of "eradication" of up to 10-15\% [34]. Future randomised controlled studies will need clear information on definitions, techniques used and timing of testing for eradication.

Consensus statement

A randomised controlled trial of $P$. aeruginosa eradication therapy, compared to no eradication treatment, should be performed.

What is the optimal antibiotic regimen (dosage, how many antibiotics, type, oral versus intravenous versus inhaled/nebulised and length of therapy) for an exacerbation of bronchiectasis?

Data evaluating the use of antibiotics during an exacerbation are extremely heterogeneous in terms of the antimicrobials used, route of administration, duration of treatment and clinical/microbiological end-points. The possibility of treating exacerbations of bronchiectasis with nebulised antibiotics has also been tested in the past [35]. Notably, there are no randomised placebo-controlled trials of antibiotic regimes during exacerbation. The appropriate length of treatment for exacerbations is also unknown, while consensus guidelines recommend 14 days of treatment with antibiotic therapy [9]. The optimal duration of treatment is important as prolonged treatment carries a higher risk of driving antibiotic side-effects, including resistance.

Consensus statement

A randomised controlled trial comparing at least 14 days of antibiotic treatment for exacerbations with shorter course treatments is required. 
What are the prevalence and characteristics of microbiological colonisation in patients with bronchiectasis across Europe lincluding bacteria, viruses, fungi, nontuberculous mycobacteria and resistant microorganisms)?

Haemophilus influenzae and P. aeruginosa are the most commonly isolated organisms in several European studies using aerobic selective cultures, although no organisms are isolated in $23-27 \%$ of patients $[9,17,36,37]$. New methods to study lung microbiota have found that the diversity of airway infection is underestimated, with anaerobic bacteria found in up to $83 \%$ of sputum samples, and that three taxa, Streptococcaceae, Pseudomonadaceae and Pasturellaceae, seem to be dominant [38]. However, most microbiome studies in bronchiectasis to date have been small, and therefore the clinical importance of this information is uncertain. Few data have been published regarding the prevalence of fungal colonisation and it is now possible to perform sequencing of the fungal "mycobiome" in a similar way to that described above for bacteria [38-40]. The prevalence of nontuberculous mycobacteria (NTM) in Europe is $<10 \%$, although there seems to be a broad geographic variation [41]. The role of NTM, between innocent colonisers or those causing chronic infection, and the predisposing factors to this need to be differentiated. There is also a paucity of data regarding the isolation of viruses and multiresistant bacteria $[4,18,29]$. The EMBARC registry is currently collecting susceptibility patterns of bacteria causing chronic infection in patients with bronchiectasis and these data will be crucial in planning further interventional studies on antibiotics at the European level [10]. Finally, it is desirable that agreed definitions of important concepts such as initial colonisation, intermittent isolation, chronic colonisation, chronic infection, eradication and exacerbation should be adopted across Europe.

\section{Consensus statements}

1) We suggest studies of the microbiome (incorporating bacteria and potentially fungi) in bronchiectasis linked to detailed clinical phenotyping data.

2) A longitudinal study of the bacteriology of bronchiectasis incorporating data on antibiotic resistance is needed.

\section{What is the impact of long-term antibiotic therapy on microbial resistances?}

The wide use of both systemic and inhaled antibiotics in patients with bronchiectasis causes rising concern about antimicrobial resistance, particularly for P. aeruginosa [42]. Since few options are currently available to intervene on microbial characteristics, most of the current efforts are dedicated to improving antibiotic characteristics and optimising their administration [43]. Regarding the use of long-term antibiotics, periodic administration of rotating or fixed antibiotics is potentially associated with increased resistance and side-effects, and risk of selection of fungal infection [38, 44, 45]. Although prolonged therapy with macrolides is effective in reducing exacerbations, there is a clear risk of antibiotic resistance for both sputum and oropharyngeal flora, and a more careful selection of patients undergoing this treatment is recommended [46-49]. The risk of antimicrobial resistance to inhaled antibiotics seems to be very low despite prolonged and continuous administration, perhaps due to the high concentrations achieved in the airways [33].

Consensus statements

1) Longitudinal studies should be conducted in patients receiving oral and inhaled antibiotics to monitor for the emergence of antibiotic resistance.

2) Studies should ideally evaluate whether cyclic or continuous administration of long-term antibiotics is superior both in terms of clinical efficacy and the emergence of resistance.

\section{When should a long-term suppressive antibiotic therapy (either oral or inhaled/nebulised) be started in patients with bronchiectasis laccording to the presence or absence of $\mathrm{P}$. aeruginosa or other pathogens) and what should be the end-points for efficacy?}

Several reports describe the long-term use of inhaled antibiotics in $\sim 10 \%$ and of macrolides in $\sim 30 \%$ of all bronchiectasis patients [50-52]. Various inhaled antibiotics have been tested to reduce bacterial load from bronchiectasis patients' airways, and related symptoms and exacerbations, such as tobramycin [53, 54], colistin [55], gentamicin [56] and aztreonam [57]. In bronchiectasis, data on inhaled antibiotics are limited and results have been mixed, with tolerability being one of the major issues. As a result of the challenges in published trials, none are as yet licensed for use in bronchiectasis by authorities in Europe or the USA.

Three different trials have largely demonstrated the usefulness of long-term macrolides in reducing the number of exacerbations with consequent improvement of quality of life and, in some cases, with slower lung function decline [46-48]. Nevertheless, it is important to remember several concerns about long-term use of macrolides, including antimicrobial resistance $[48,58]$, the potential to promote macrolide-resistant NTM [59-61] and an increased risk of cardiovascular complications [62]. It seems that macrolides are clearly beneficial in patients with bronchiectasis but the optimal patient population to benefit has not been defined. 
The inclusion criteria of the trials were broad and each trial used a different regimen. Trials had either 6-month [46] or 12-month $[47,48]$ treatment duration, and the long-term safety and resistance impact of these drugs is unknown.

Consensus statements

1) Further studies are required to define the optimal patient population to benefit from long-term macrolide therapy.

2) More "real world" data on the long-term safety and resistance impact of macrolide treatment are required.

3) Inhaled antibiotics such as colistin, gentamicin and tobramycin should be subject to definitive phase III trials to demonstrate a reduction in exacerbations and improvements in quality of life.

What are the key factors leading to P. aeruginosa colonisation?

The reason why some patients with bronchiectasis become colonised with $P$. aeruginosa while the majority do not is unexplained. Genetic studies may identify host risk factors for $P$. aeruginosa colonisation, and a modest effect of mannose-binding lectin polymorphisms on susceptibility has been shown in both bronchiectasis and CF [63, 64]. Microbial factors are also important in $P$. aeruginosa colonisation, with person-person transmission and epidemic strains being well described in CF but not in non-CF bronchiectasis [65]. Furthermore, different $P$. aeruginosa strains in $\mathrm{CF}$ have been recognised to have variation in in vitro phenotypes that appear to translate into clinically meaningful outcomes [66, 67].

\section{Consensus statements}

1) Mechanistic studies investigating the genetic, microbiological, inflammatory and clinical susceptibility factors for $P$. aeruginosa colonisation should be conducted.

2) Long-term cohort studies are needed to identify which patients acquire P. aeruginosa colonisation and to identify its independent effects on outcome.

What are the indications of oral versus inhaled/nebulised long-term suppressive antibiotic treatment?

There are no head-to-head trials of oral versus inhaled antibiotics. The criteria to choose between oral macrolides and inhaled antibiotics are still not clear; the decision is still empirical, and based on personal experience and local healthcare prescription rules. Nevertheless, it is clear that some factors could justify the antibiotic choice, such as the presence of specific antibiotic allergies and side-effects, the patients' preferences and ability to manage inhalations, the co-existence of rhinosinusitis, and cardiovascular comorbidities. In the absence of head-to-head trials, large registries should provide important information about treatment patterns [10]. In addition, ongoing randomised trials of inhaled antibiotics that include macrolide-treated patients will evaluate the important question of whether inhaled antibiotics can provide added benefit.

\section{Consensus statement}

Comparative studies are needed to determine the optimal choice between oral and inhaled antibiotic treatment in patients with and without $P$. aeruginosa colonisation.

What are the best molecule, dose, regimen and duration for long-term oral antibiotic therapy in patients with bronchiectasis laccording to the presence or absence of $\mathrm{P}$. aeruginosa or other pathogens)?

Three major studies recently demonstrated the efficacy of long-term macrolides in bronchiectasis in double-blind randomised trials [46-48]. Key questions remain regarding oral antibiotic therapy including: do macrolides have to be continued lifelong or can they be withdrawn (e.g. after 12 months)? The most appropriate dose and macrolide agent to minimise side-effects and development of antimicrobial resistance has not been determined. It is not known if alternative oral antibiotic agents such as tetracyclines or $\beta$-lactams are equally effective when given long term. As the maximum duration of macrolide treatment was 12 months, it is not known if the effectiveness of macrolides wanes over time, as antibiotic resistance develops or if effectiveness is sustained.

Consensus statement

Randomised controlled trials should address whether alternative long-term oral antibiotics (other than macrolides) are effective at reducing exacerbations. 
Important research priorities identified by patients

Other important themes were identified from the top-ranking patient priorities, with special attention focused on condition management, communication and information. These areas were all strongly supported by the expert working group.

\section{Condition management}

The questionnaire identified a number of research topics that could help improve the management of their bronchiectasis. $>96 \%$ of respondents felt that their bronchiectasis could be better managed through having a self-management plan co-designed with their HCP, and access to physiotherapy/pulmonary rehabilitation, which also includes teaching them how to use techniques/equipment at home [1, 22, 40, 68, 69]. Self-management plans facilitated by good communication between patients and HCPs empower patients to manage and cope with their condition more confidently and independently [70, 71]. An important component of these self-management strategies and of reducing hospitalisation is the awareness of HCPs of bronchiectasis and available and appropriate community care and physiotherapy services $[1,68,72]$.

\section{Consensus statement}

Studies should be conducted to determine the effectiveness of patient self-management in bronchiectasis and adherence to treatment.

\section{Communication and information}

One of the top priorities for patients was good communication between HCPs and each patient. Patients also ranked highly the need for access to reliable, plain-language information on living with bronchiectasis [70]. This shows that patients do not feel their information needs are being met, as they are struggling to find accurate information to help them live with their condition, which is a role that can be supported by the clear communication of information to patients by HCPs, both at the point of diagnosis and as their condition/needs change [70]. Increasingly, patients look to the internet for information on their condition; therefore, HCPs can provide an invaluable service by signposting patients and their carers/families to reliable plain language information both online and in paper format, i.e. medically accurate, plain-language information leaflets. This role can be especially important for people with bronchiectasis, and other neglected and under-resourced conditions, where there is less public and HCP awareness, and few widely available multilingual information leaflets, patient organisations and support groups. The potential for enhanced information packages or patient alert systems to help adherence and self-management offers a potentially cost effective solution acceptable to patients, with examples available in other disease areas and with patients involved in the development of resources.

\section{Consensus statements}

1) Further research with patients as partners could explore the specific information needs of bronchiectasis patients, and effective HCP and patient communication strategies, and develop improved patient-reported outcomes.

2) A multidisciplinary education programme is needed for bronchiectasis to increase awareness among nonspecialists in secondary care and in primary care.

Priorities identified in this document should be important to inform the work of individual researchers, the EMBARC network as a whole, charities, funding bodies, regulators and healthcare policy makers. The EMBARC steering committee and the international advisory board (www.bronchiectasis.eu) unanimously approved the present document and research priorities with the aim of supporting studies designed to answer these questions.

A key finding of our consensus process is that very few of our research questions can be answered by pure basic or clinical research and almost all of these priorities require an integrated approach with careful clinical phenotyping, as will be available in multinational registries, linked to translational and mechanistic research. It is well recognised that there is a barrier between basic and clinical researchers leading to a "translation gap". As a result of this process, we propose that a key objective of the EMBARC CRC should be to promote collaboration between clinical and scientific researchers in the field of bronchiectasis.

Our selection of the most important research priorities herein does not imply that other research questions are not also important. All of the 55 priorities identified by the Delphi process and all of the priorities assessed by patients were rated as important by the majority of questionnaires. Furthermore, patients reported different priorities specifically based on their daily experience of and concerns about the future of their bronchiectasis. This does not preclude that other signs/symptoms, such as weight loss or depression, are not crucial, with a relevant impact on outcomes. 
One of the limitations of this project for the patients' questionnaire is that we used pre-worded and multiple-choice questions, rather than running one-to-one interviews or focus groups, potentially missing the complexity of bronchiectasis patients' experiences. However, our questionnaire seems to be the best method to rapidly reach the largest sample size of patients with bronchiectasis across Europe expressing the patients' perspective on "knowledge gaps", and it is the largest such endeavour published so far. Another limitation is the absence of the involvement among experts (mainly physicians running bronchiectasis clinics) of other HCPs taking care of bronchiectasis patients, such as general practitioners. Their presence would have led to a change in research priorities, probably in favour of nonantibiotic treatments. Finally, we should acknowledge that $40 \%$ of respondents to the patient questionnaires were people from the UK and this finding could slightly affect the generalisability of patients' priorities across Europe. However, the pragmatic methodology we used in this project facilitated insight into an under-researched population, especially in terms of patient-reported needs, and a health issue that affects patients and services across Europe. Although response rates differed by language/country and disease, we should recognise that the sample did share key characteristics with the European respiratory patient population.

One of the strengths of this document is the comparison, for the first time in literature, of both experts' and patients' perspectives in bronchiectasis research in order to point out their shared research priorities. Furthermore, the voting process for both experts and patients was performed anonymously.

\section{Conclusions}

This consensus statement identifies the key research priorities as determined by physicians caring for bronchiectasis patients, by the patients themselves, and by the friends and family helping care for them. This document will be a valuable resource for public and private stakeholders involved in designing calls for research funding at both national and international level. The EMBARC initiative seeks to stimulate clinical and translational research in bronchiectasis, and the priorities identified here provide the clearest possible roadmap towards improving our understanding of the disease and the quality of care for bronchiectasis patients.

\section{Acknowledgements}

The EMBARC Study Group (including the International Advisory Board) investigators are: Francesco Blasi (University of Milan, IRCCS Fondazione Ospedale Maggiore, Milan, Italy), Wim Boersma (Medical Centre Alkmaar, Alkmaar, the Netherlands), Katerina Dimakou (Chest Diseases Hospital, Athens, Greece), Stuart Elborn (Centre for Infection and Immunity, Belfast, UK), Charles Haworth (Papworth Hospital, Cambridge, UK), Adam Hill (Royal Infirmary of Edinburgh, Edinburgh, UK), Marlène Murris-Espin (Hopital Larrey-CHU de Toulouse, Toulouse, France), Antoni Torres (Hospital Clinic, Barcelona, Spain), Robert Wilson (Royal Brompton Hospital, London, UK), Tobias Welte (Medizinische Hochschule Hanover, Hanover, Germany), Timothy Aksamit (Mayo Clinic College of Medicine, Rochester, MN, USA), Charles Feldman (Charlotte Maxeke Johannesburg Academic Hospital, and Faculty of Health Sciences University of the Witwatersrand, Johannesburg, South Africa) and Oscar Rizzo (Asociación Argentina de Medicina Respiratoria, Buenos Aires, Argentina).

We would like to thank the patient advisory group who reviewed the questionnaire and input into the EMBARC meetings that helped shape the development of this project, and to the respondents for sharing their experiences and research priorities via the questionnaire.

\section{References}

1 Chalmers JD, Aliberti S, Blasi F. Management of bronchiectasis in adults. Eur Respir J 2015; 45: 1446-1462.

2 Ringshausen FC, de Roux A, Pletz MW, et al. Bronchiectasis-associated hospitalizations in Germany, 2005-2011: a population-based study of disease burden and trends. PLoS One 2013; 8: e71109.

3 Seitz AE, Olivier KN, Adjemian J, et al. Trends in bronchiectasis among Medicare beneficiaries in the United States, 2000 to 2007. Chest 2012; 142: 432-439.

4 Chalmers JD, Goeminne P, Aliberti S, et al. The bronchiectasis severity index. An international derivation and validation study. Am J Respir Crit Care Med 2014; 189: 576-585.

5 Loebinger MR, Wells AU, Hansell DM, et al. Mortality in bronchiectasis: a long-term study assessing the factors influencing survival. Eur Respir J 2009; 34: 843-849.

6 Goeminne PC, Nawrot TS, Ruttens D, et al. Mortality in non-cystic fibrosis bronchiectasis: a prospective cohort analysis. Respir Med 2014; 108: 287-296.

7 Quint JK, Millett ER, Joshi M, et al. Changes in the incidence, prevalence and mortality of bronchiectasis in the UK from 2004 to 2013: a population-based cohort study. Eur Respir J 2016; 47: 186-193.

8 Ringshausen FC, de Roux A, Diel R, et al. Bronchiectasis in Germany: a population-based estimation of disease prevalence. Eur Respir J 2015; 46: 1805-1807.

9 Pasteur MC, Bilton D, Hill AT. British Thoracic Society guideline for non-CF bronchiectasis. Thorax 2010; 65: Suppl. 1: i1-i58.

10 Chalmers JD, Aliberti S, Polverino E, et al. The EMBARC European Bronchiectasis Registry: protocol for an international observational study. ERJ Open Res 2015; 1: 00081-2015.

11 Wedzicha W, Fletcher M, Powell P. Making ERS guidelines relevant and accessible: involving patients and the public. Breathe 2011; 8: 9-11.

12 Murphy MK, Black NA, Lamping DL, et al. Consensus development methods, and their use in clinical guideline development. Health Technol Assess 1998; 2: 1-88. 
13 Lonni S, Chalmers JD, Goeminne PC, et al. Etiology of non-cystic fibrosis bronchiectasis in adults and its correlation to disease severity. Ann Am Thorac Soc 2015; 12: 1764-1770.

14 Fodor AA, Klem ER, Gilpin DF, et al. The adult cystic fibrosis airway microbiota is stable over time and infection type, and highly resilient to antibiotic treatment of exacerbations. PLoS One 2012; 7: e45001.

15 Han MK, Huang YJ, Lipuma JJ, et al. Significance of the microbiome in obstructive lung disease. Thorax 2012; 67: 456-463.

16 Sethi S, Murphy TF. Infection in the pathogenesis and course of chronic obstructive pulmonary disease. $N$ Engl $J$ Med 2008; 359: 2355-2365.

17 Tunney MM, Einarsson GG, Wei L, et al. Lung microbiota and bacterial abundance in patients with bronchiectasis when clinically stable and during exacerbation. Am J Respir Crit Care Med 2013; 187: 1118-1126.

18 Gao Y, Guan W, Xu G, et al. The role of viral infection in pulmonary exacerbations of bronchiectasis in adults: a prospective study. Chest 2015; 147: 1635-1643.

19 Goeminne PC, Bijnens E, Nemery B, et al. Impact of traffic related air pollution indicators on non-cystic fibrosis bronchiectasis mortality: a cohort analysis. Respir Res 2014; 15: 108.

20 Lee AL, Burge A, Holland AE. Airway clearance techniques for bronchiectasis. Cochrane Database Syst Rev 2013; 5: CD008351.

21 Ong HK, Lee AL, Hill CJ, et al. Effects of pulmonary rehabilitation in bronchiectasis: a retrospective study. Chron Respir Dis 2011; 8: 21-30.

22 Mandal P, Sidhu MK, Kope L, et al. A pilot study of pulmonary rehabilitation and chest physiotherapy versus chest physiotherapy alone in bronchiectasis. Respir Med 2012; 106: 1647-1654.

23 Lee AL, Hill CJ, Cecins N, et al. The short and long term effects of exercise training in non-cystic fibrosis bronchiectasis- a randomised controlled trial. Respir Res 2014; 15: 44.

24 Martínez-García MÁ, de Gracia J, Vendrell Relat M, et al. Multidimensional approach to non-cystic fibrosis bronchiectasis: the FACED score. Eur Respir J 2014; 43: 1357-1367.

25 Hayes D Jr, Kopp BT, Tobias JD, et al. Survival in patients with advanced non-cystic fibrosis bronchiectasis versus cystic fibrosis on the waitlist for lung transplantation. Lung 2015; 193: 933-938.

26 Grillo L, Irving S, Hansell DM, et al. The reproducibility and responsiveness of the lung clearance index in bronchiectasis. Eur Respir J 2015; 46: 1645-1653.

27 Aliberti S, Lonni S, Dore S, et al. Clinical phenotypes in adult patients with bronchiectasis. Eur Respir J 2016; 47: 1113-1122.

28 Finch S, McDonnell MJ, Abo-Leyah H, et al. A comprehensive analysis of the impact of Pseudomonas aeruginosa colonisation on prognosis in adult bronchiectasis. Ann Am Thorac Soc 2015; 12: 1602-1611.

29 McDonnell MJ, Jary HR, Perry A, et al. Non cystic fibrosis bronchiectasis: a longitudinal retrospective observational cohort study of Pseudomonas persistence and resistance. Respir Med 2015; 109: 716-726.

30 Langton Hewer SC, Smyth AR. Antibiotic strategies for eradicating Pseudomonas aeruginosa in people with cystic fibrosis. Cochrane Database Syst Rev 2014; 11: CD004197.

31 White L, Mirrani G, Grover M, et al. Outcomes of Pseudomonas eradication therapy in patients with non-cystic fibrosis bronchiectasis. Respir Med 2012; 106: 356-360.

32 Orriols R, Hernando R, Ferrer A, et al. Eradication therapy against Pseudomonas aeruginosa in non-cystic fibrosis bronchiectasis. Respiration 2015; 90: 299-305.

33 Brodt AM, Stovold E, Zhang L. Inhaled antibiotics for stable non-cystic fibrosis bronchiectasis: a systematic review. Eur Respir J 2014; 44: 382-393.

34 Wilson R, Welte $\mathrm{T}$, Polverino E, et al. Ciprofloxacin dry powder for inhalation in non-cystic fibrosis bronchiectasis: a phase II randomised study. Eur Respir J 2013; 41: 1107-1115.

35 Bilton D, Henig N, Morrissey B, et al. Addition of inhaled tobramycin to ciprofloxacin for acute exacerbations of Pseudomonas aeruginosa infection in adult bronchiectasis. Chest 2006; 130: 1503-1510.

36 Chalmers JD, Smith MP, McHugh BJ, et al. Short- and long-term antibiotic treatment reduces airway and systemic inflammation in non-cystic fibrosis bronchiectasis. Am J Respir Crit Care Med 2012; 186: 657-665.

37 Purcell P, Jary H, Perry A, et al. Polymicrobial airway bacterial communities in adult bronchiectasis patients. BMC Microbiol 2014; 14: 130.

38 Maiz L, Vendrell M, Olveira C, et al. Prevalence and factors associated with isolation of Aspergillus and Candida from sputum in patients with non-cystic fibrosis bronchiectasis. Respiration 2015; 89: 396-403.

39 Angrill J, Agustí C, de Celis R, et al. Bacterial colonisation in patients with bronchiectasis: microbiological pattern and risk factors. Thorax 2002; 57: 15-19.

40 Pasteur MC, Helliwell SM, Houghton SJ, et al. An investigation into causative factors in patients with bronchiectasis. Am J Respir Crit Care Med 2000; 162: 1277-1284.

41 Bonaiti G, Pesci A, Marruchella A, et al. Nontuberculous mycobacteria in noncystic fibrosis bronchiectasis. Biomed Res Int 2015; 2015: 197950.

42 Hawkey PM. Mechanisms of quinolone action and microbial response. J Antimicrob Chemother 2003; 51: Suppl. 1, 29-35.

43 Tay GT, Reid DW, Bell SC. Inhaled antibiotics in cystic fibrosis (CF) and non-CF bronchiectasis. Semin Respir Crit Care Med 2015; 36: 267-286.

44 Prolonged antibiotic treatment of severe bronchiectasis; a report by a subcommittee of the Antibiotics Clinical Trials (Non-Tuberculous) Committee of the Medical Research Council. Br Med J 1957; 2: 255-259.

45 Evans DJ, Bara AI, Greenstone M. Prolonged antibiotics for purulent bronchiectasis in children and adults. Cochrane Database Syst Rev 2007; CD001392.

46 Wong C, Jayaram L, Karalus N, et al. Azithromycin for prevention of exacerbations in non-cystic fibrosis bronchiectasis (EMBRACE): a randomised, double-blind, placebo-controlled trial. Lancet 2012; 380: 660-667.

47 Altenburg J, de Graaff CS, Stienstra Y, et al. Effect of azithromycin maintenance treatment on infectious exacerbations among patients with non-cystic fibrosis bronchiectasis: the BAT randomized controlled trial. JAMA 2013; 309: 1251-1259.

48 Serisier DJ, Martin ML, McGuckin MA, et al. Effect of long-term, low-dose erythromycin on pulmonary exacerbations among patients with non-cystic fibrosis bronchiectasis: the BLESS randomized controlled trial. JAMA 2013; 309: 1260-1267. 
49 Serisier DJ. Risks of population antimicrobial resistance associated with chronic macrolide use for inflammatory airway diseases. Lancet Respir Med 2013; 1: 262-274.

50 Nicotra $\mathrm{MB}$, Rivera M, Dale $\mathrm{AM}$, et al. Clinical, pathophysiologic, and microbiologic characterization of bronchiectasis in an aging cohort. Chest 1995; 108: 955-961.

51 Davies G, Wells AU, Doffman S, et al. The effect of Pseudomonas aeruginosa on pulmonary function in patients with bronchiectasis. Eur Respir J 2006; 28: 974-979.

52 Hill AT, Welham S, Reid K, et al. British Thoracic Society national bronchiectasis audit 2010 and 2011. Thorax 2012; 67: 928-930.

53 Barker AF, Couch L, Fiel SB, et al. Tobramycin solution for inhalation reduces sputum Pseudomonas aeruginosa density in bronchiectasis. Am J Respir Crit Care Med 2000; 162: 481-485.

54 Drobnic ME, Suñé P, Montoro JB, et al. Inhaled tobramycin in non-cystic fibrosis patients with bronchiectasis and chronic bronchial infection with Pseudomonas aeruginosa. Ann Pharmacother 2005; 39: 39-44.

55 Haworth CS, Foweraker JE, Wilkinson P, et al. Inhaled colistin in patients with bronchiectasis and chronic Pseudomonas aeruginosa infection. Am J Respir Crit Care Med 2014; 189: 975-982.

56 Murray MP, Govan JR, Doherty CJ, et al. A randomized controlled trial of nebulized gentamicin in non-cystic fibrosis bronchiectasis. Am J Respir Crit Care Med 2011; 183: 491-499.

57 Barker AF, O'Donnell AE, Flume P, et al. Aztreonam for inhalation solution in patients with non-cystic fibrosis bronchiectasis (AIR-BX1 and AIR-BX2): two randomised double-blind, placebo-controlled phase 3 trials. Lancet Respir Med 2014; 2: 738-749.

58 Rogers GB, Bruce KD, Martin ML, et al. The effect of long-term macrolide treatment on respiratory microbiota composition in non-cystic fibrosis bronchiectasis: an analysis from the randomised, double-blind, placebo-controlled BLESS trial. Lancet Respir Med 2014; 2: 988-996.

59 Renna M, Schaffner C, Brown K, et al. Azithromycin blocks autophagy and may predispose cystic fibrosis patients to mycobacterial infection. J Clin Invest 2011; 121: 3554-3563.

60 Aksamit TR, Philley JV, Griffith DE. Nontuberculous mycobacterial (NTM) lung disease: the top ten essentials. Respir Med 2014; 108: 417-425.

61 Coolen N, Morand P, Martin C, et al. Reduced risk of nontuberculous mycobacteria in cystic fibrosis adults receiving long-term azithromycin. J Cyst Fibros 2015; 14: 594-599.

62 Schembri S, Williamson PA, Short PM, et al. Cardiovascular events after clarithromycin use in lower respiratory tract infections: analysis of two prospective cohort studies. BMJ 2013; 346: f1235.

63 Chalmers JD, McHugh BJ, Doherty C, et al. Mannose-binding lectin deficiency and disease severity in non-cystic fibrosis bronchiectasis: a prospective study. Lancet Respir Med 2013; 1: 224-232.

64 Chalmers JD, Fleming GB, Hill AT, et al. Impact of mannose-binding lectin insufficiency on the course of cystic fibrosis: a review and meta-analysis. Glycobiology 2011; 21: 271-282.

65 Fothergill JL, Walshaw MJ, Winstanley C. Transmissible strains of Pseudomonas aeruginosa in cystic fibrosis lung infections. Eur Respir J 2012; 40: 227-238.

66 Al-Aloul M, Crawley J, Winstanley C, et al. Increased morbidity associated with chronic infection by an epidemic Pseudomonas aeruginosa strain in CF patients. Thorax 2004; 59: 334-336.

67 Fothergill JL, Mowat E, Ledson MJ, et al. Fluctuations in phenotypes and genotypes within populations of Pseudomonas aeruginosa in the cystic fibrosis lung during pulmonary exacerbations. J Med Microbiol 2010; 59: $472-481$.

68 O'Donnell AE. Bronchiectasis. Chest 2008; 134: 815-823.

69 Zanini A, Aiello M, Adamo D, et al. Effects of pulmonary rehabilitation in patients with non-cystic fibrosis bronchiectasis: a retrospective analysis of clinical and functional predictors of efficacy. Respiration 2015; 89: 525-533.

70 Hester K, McAlinden P, De Soyza A. Education and information for patients with bronchiectasis: what do patients want? Eur Respir J 2011; 38: 3622.

71 McCullough A, Tunney MM, Elborn JS, et al. All illness is personal to that individual: a qualitative study of patients' perspectives on treatment adherence in bronchiectasis. Health Expectations 2015; 18: 2477-2488.

72 Baggott CJ, Harris E, Suntharalingam J, et al. Non CF bronchiectasis: smoothing the process: clinical management of COPD and bronchiectasis. Thorax 2014; 69: A118-A119. 